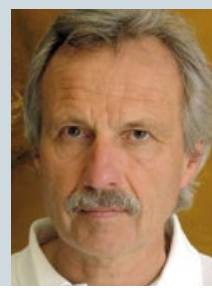

Dr. med. Michael

Pieper

Facharzt für

orthopädische

Rheumatologie

und Handchirurgie,

Endocenter

Damme

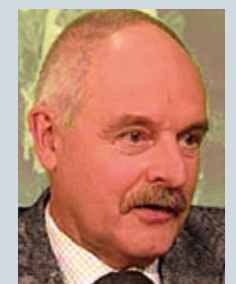

Prof. Dr. Klaus A. Milachowski

Orthopädische

Privatpraxis,

Belegarzt an der

Klinik Dr. Schreiber,

München

\section{Initiale Kombitherapie von Vorteil bei ACPA-positiver rheumatoider Arthritis}

\author{
Patienten mit rheumatoider Arthritis (RA) und Antikörpern gegen citrullinierte \\ Peptide (ACPA) sprechen klinisch auf unterschiedliche Therapiestrategien \\ vergleichbar gut an wie solche ohne ACPA. Allerdings scheint bei positivem \\ ACPA-Status eine initiale Kombitherapie besser geeignet, um Gelenkschäden \\ zu vermeiden.
}

A CPA-positive RA-Patienten gelangen bekanntlich schwerer in Remission, zeigen eine höhere Krankheitsaktivität und entwickeln häufiger Gelenkschäden als ACPA-negative. Es wird daher oft spekuliert, dass es sich bei ACPApositiver und ACPA-negativer RA um zwei verschiedene Erkrankungen handelt, bei denen die Patienten demzufolge auch unterschiedlich behandelt werden müssten. In einer Subgruppenanalyse der vierarmigen Studie BeSt (Behandlungs-Strategien) bei über 500 RA-Patienten konnte ein Team niederländischer Wissenschaftler um Dr. Marianne van den Broek nun aber keine Hinweise darauf finden, dass ACPA-Positive und ACPA-Negative unter klinischen Gesichtspunkten eine andere Therapiestrategie benötigen, wohl aber unter radiologischen Aspekten.

In der Studie analysierten die Rheumatologen über acht Jahre hinweg die Progression von Gelenkschäden, die Zeit der klinischen Remission, die Dauer einer medikamentenfreien Remission, sowie körperliche Einschränkungen. Zudem bestimmten sie regelmäßig den $\mathrm{Di}$ sease Activity Score 44 (DAS). Die Patienten wurden auf vier Arme verteilt: Eine Gruppe erhielt eine sequenzielle Monotherapie mit Methotrexat (MTX), die zweite Gruppe eine Step-up-KombiDie dritte Gruppe bekam eine initiale Kombinationstherapie aus Basistherapeutika und Prednison und die vierte Gruppe eine initiale Kombination des TNF-alfa-Blockers Infliximab mit MTX. Alle drei Monate wurde die Krankheitsaktivität mithilfe des DAS bestimmt. Bei mehr als 2,4 Punkten wurde die Therapie angepasst.

Das Ergebnis: Die Ansprechraten von ACPA-positiven und -negativen Patienten waren in allen Therapiearmen ähnlich gut. Im Schnitt erreichten die Patienten nach einem Jahr einen DAS-Wert von unter 2,0. Die Veränderungen im DAS waren in beiden Gruppen vergleichbar. Auch der Anteil der Patienten, die mindestens eine Remission pro Jahr erreichten, hing nicht vom Antikörperstatus ab. Wie erwartet, kam es bei ACPA-Positiven insgesamt aber häufiger zu einer Progression der Gelenkschäden, vor allem bei initialer MTX-Monotherapie, und die RA flackerte nach einer Remission häufiger wieder auf als bei ACPA-Negativen.

Fazit: Bei der Wahl der Therapie sollten sich Rheumatologen auch weiterhin primär am DAS-Wert orientieren. Für ACPA-positive Patienten scheint jedoch eine initiale MTX-Therapie nicht zu ge- nationstherapie mit Basistherapeutika.

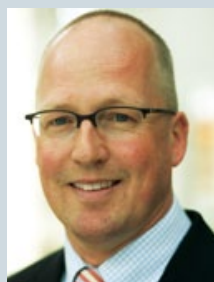

Dr. med. Stefan Middeldorf

Chefarzt

Orthopädische

Klinik,

Schön Klinik,

Bad Staffelstein nügen, um Gelenkschäden zu verhindern. Die Studie liefert daher ein weiteres Argument, bei solchen Patienten mit einer Kombitherapie zu beginnen.

Thomas Müller

Van den Broek $M$ et al. The association of treatment response and joint damage with ACPA-status in recent-onset RA: a subanalysis of the 8-year follow-up of the BeSt study. Ann Rheum Dis 2012;71:245-248.

Kommentar: Aus den Daten der BeST-Studie wurden bereits wichtige Aspekte hinsichtlich einer optimalen Behandlungsstrategie bei Patienten mit rheumatoider Arthritis abgeleitet. Dies setzt sich mit der aktuell vorliegenden Publikation zum langfristigen Therapieansprechen bei ACPA-positiven und ACPA-negativen RA-Patienten fort. Die Auswertungen zeigen, dass sich hinsichtlich des klinischen Ansprechens keine relevanten Unterschiede zwischen Patienten mit, respektive ohne ACPA in den verschiedenen Therapiearmen nachweisen lassen. Allerdings bestätigt die Analyse der radiologischen Daten den bekanntermaßen aggressiveren radiologischen Verlauf der RA bei den ACPA-positiven Patienten. Der ACPA-Status ist deshalb in der klinischen Praxis als wichtiger Prognosefaktor einzuordnen, der die Entscheidung für eine initiale Kombinationstherapie bei einzelnen RA-Patienten mit schlechter Krankheitsprognose unterstützt.

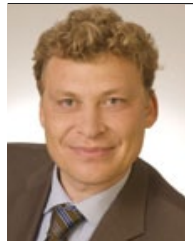

Prof. Dr. med. Martin Fleck Klinik und Poliklinik für Innere Medizin I, Universitätsklinikum 93042 Regensburg und Klinik für Rheumatologie/Klinische Immunologie, Asklepios-Klinikum Kaiser-Karl-V.-Allee 3, 93077 Bad Abbach E-Mail: martin.fleck@klinik.uni-r.de 